\title{
The Theory of Aesthetic Characteristics of Chinese Court Dance
}

\author{
Yanbin Ding ${ }^{1}$ \\ ${ }^{1}$ Yuxi Normal University, Yunnan Yuxi ,China, 653100 \\ Dingyanbin66@126.com
}

Keywords: The Palace Dance; Aesthetic Characteristics; To Fly through God; Feathers in the Wind; The Actual Interactio; Sleeve To Spring Willow

\begin{abstract}
In the ancient Chinese art, poetry, music and dance, and then gradually produces the differentiation, the formation of the distinctive arts, and the palace dance with its reigns for the lofty status and economic power, focused mainly on folk dance dance in all walks of life essence for its political and hedonic service, at the same time, the court of the professional dance and relying on its excellent artistic talents to create high quality dance. After a long period of precipitation, the palace dance naturally become the symbol of Chinese civilization for thousands of years dance, its content is extensive, forms of diversity, characteristics of bright, reveals the palace dance for us to fly through god, ethereal and rhythm, feathered from wind, lightsome and detachment, the actual interactions - time and space and sleeve, sleeve spring willow - dance count four aesthetic characteristics.
\end{abstract}

\section{Introduction}

Chinese court dance is a part of Chinese history and culture, as a result, the variation and development of Chinese history and culture in different stages of the, directly affects the content and form of dance, palace due to various historical stages of economic, political, ideological, social life, thus determines the Chinese court dance at each stage of development and the characteristics of the aesthetic, such as the western zhou dynasty gagaku ZhuangSu lofty, han palace dance potential zhang bo, yan music in the tang dynasty colorful, yuan palace dance diverse interest such as radiation with different aesthetic characteristics in different dynasties. Indeed, a strong vitality, are in the spirit of "dao" as its inner core, to "artistic conception" as the duality of internal and external emotion, internal harmony of the universe with the liquidity and revelation of variability and rhythm, as Mr Zong white said: "' way of life and the life of" art ", in virtual, alto before and suitable for xianchi \&jingshou dance, is the back of the first hitches. Music rhythm is that they frame." [1] "dance" is the height of the rhythm, rhythm, order, rationality, at the same time is the most direct high vitality, the most essential and the most hot and good performance. More than 4000 years history of Chinese court dance, in its long development, again and again to create a brilliant, roots, generally exist the following aesthetic characteristics.

\section{To Fly God-Ethereal And Rhythm}

Witchcraft dancing is very developed in ancient China, especially in shang dynasty, in the courts of shang dynasty, almost all the major religious witchcraft activities are accompanied by shamanistic witch dance, these "witch" is god, and god's spokesperson, witch dance movements form must as far as possible the will of god and the form of god. In wrath of the dance of the whole process, in addition to the peculiar charm, frantic calls, shock people, with the melody of the abnormal shape on the show, the action of madness, have a kind of to leap meeting with god's ethereal dancing, embodies the spirit of flying.

"Dragon" as a symbol of the Chinese nation, its image is a deer horn, dog, fish scales and must be, full of curves and rhythm on modelling, it is free, dancing foot claws, those taking the special weather, as a ritual dance, with a somewhat mysterious strange and somewhat naive, one of the most amazing, to "gas", show the external most give fantasy sports, that is "fly". 
The tang dynasty is the development of Chinese ancient dance on a glorious period, its prosperity is largely depends on the background of the society at that time. The tang dynasty is in great turmoil in relative to break the feudal autocracy, far from the latter's neo-confucianism era, new cultural norms to establish the transition stage. On ideology, the rulers of Confucianism, Buddhism, Taoism three ideas keep a hybrid compatible attitude, contributed to the relative instability of The Times, gives people give full play to their creativity and talent of freedom and opportunity, it is in this loose the cultural environment of unprecedented development, formed the buddhist flying the ci-poetry of net world. Flying the ci-poetry music dance acting aesthetic feeling is the strongest image, its volley soar, its body flex, twist, the acting fantasy, super hard, and not the human body; Its roaring, plankton, Banks, wiggle gesture, is beautiful to the extreme, roaring wave, in the bustle of completely forgotten in the dizzying, enjoy the happiness of the kingdom of heaven, as $\mathrm{Mr}$ Tan Shutong in the dunhuang flying art movement of study summarizes its five characteristics: "the rhythm of the imposing manner of flying, flying, flying interest, taking the pen and ink, and the change of the flying". [2] therefore, changeful dunhuang flying apsaras flying is the life and soul of art, is the momentum and vigor of life through heaven and earth reiki, it fully shows the burst all spirit of freedom, and to "fly" momentum throughout, in order to show a kind of freedom, elegant spirit, showing the full, with youthful vitality of passion and imagination.

\section{Feathers From Wind, Lightsome And Detachment}

The palace dance exercise to the body posture is lightsome, do one of the basic requirements, also show adequately dance skills. Jump in the spring and autumn period and the warring states period in the history of the collection of feather, the dust a travelling XuanJuan and Mo, light can mess with dust, and their body is like a feather in the wind. Have the body can do light ZhangShangWu of zhao, "floating revolving back to clear snow" Yang high-ranked imperial concubine, all embody the incomparable superb dance skills and lightsome, the aesthetic form of detachment.

Spend palace dance is right "light of advocating, there is a record of poetry:

Light body xu yang-yang he, holding his hands white cheung.

- jin "white Zhu dance"

If looks like the moon cloud river, body wave breeze turn flow.

- Liu Shuo "white Zhu dance"

Light body like without bone, the viewer is god.

Liu yuxi, the view extension branches dance 2 first"

Thin body light back to the snow, luo coagulate fat.

- bai juyi "young willows twenty rhyme"

Jiao tome evil spirit, willow lightsome body must state.

Wonderful dance fluttering, handheld light are investigated.

- sushi "USES"

Although these lines shown just dance lightly, gracefully, but obvious to let a person feel the body contains brush. Especially the white Zhu dance, because it was a time of great turmoil, many poets in the upper class for the shortness of life, eager to lengthen your life, and then hope immortal, "white Zhu dance" in the form of the original communicate people want out of this world, fly into the sky, into immortal of emotion. Indeed, with the help of the light craft, the dance can be transmitted out elegant, detachment, interest and graceful emotion and create a "feather's fairy" intent, palace dance also adapted to the emperors in the dance of unconscious on the aesthetic, in the light has reached a high level of technology.

\section{Actual Situation Interaction, Time And Space}

Time and space is the form of material existence, is also in the form of art. People sense of time is very easy to understand, from birth, aging, illness, and death of the person can feel the concept of time, so has in the past, present and future. Spatial awareness at the beginning of the physical 
fangyuan people occupied area of a kind of intuition on the basis of a priori form, we call it the perceptual space, and there's a psychological sense of space, is through the visual, tactile, kinesthetic, somatosensory senses the medium. In China's traditional thought, the art works as self-content universe as a whole, the work space is the space of artistic image to survive, "empty", "solid" the two opposite each other and complement each other, interactive form. In the opera dance, virtual sex, it is one of the most important aesthetic characteristics of Chinese opera dance. Chinese opera art is deeply rooted in Chinese traditional culture, the pursuit of "the actual phase", "and not" aesthetic realm. Chinese opera virtual sex first refers to the stage scenery and props, often to a desk, two chairs under the different scenarios, performed by actors in the different action to show a different environment, or create a mountain, water, cabinet, floor, etc. Opera is dancing and stylized movements virtual, as stage image, is the combination of virtual and real. Such as opera in horseback riding, the horse is virtual, whip props is real. Water in the boat is virtual, OARS is real. Drama of the scheduling of time and the stage is more a lot of virtual sex, stage shows and not the objective time and space of the real world, but by the actor's movements to express the plot to show at a specific time and space, make the drama stage shows a high degree of flexibility and freedom. If use the human "the peony pavilion", dreams, the common combination of multiple space of the grave to show worldly desire for the beauty of youth, the love theme, as dreams show girl was chasing wing of good love, to show after the death of the grave to good love a perennial obsession, knitting a pair of stereo multi-level, begets more scenes of the actual situation, spatio-temporal crisscross the arts scene.

\section{Sleeve Spring Willow-Dance Sleeve And Count}

Waist to the palace dance, is also an important factor that cannot be ignored, "king ChuLing good waist, palace many hungry people". Chinese court dance often dancers waist fine soft as the standard of beauty. Emperor gaozu Liu Bangzhi wife Mrs Qi is famous for "dancing eagerly count", which was to kill; Han into the emperor later zhao swallow is also beautiful, waist soft dancing by emperor in the palace. "Zhao swallow so" cloud: "zhao back bone slender, especially good yu walk, bouncing, others could not learn also". Waist is widely viewed as a dance aesthetic concerns, there are poems syndrome:

Red lead brushed face waist, golden rust stripping soft body.

- Zhang Hu Li Guta branch,

Waist for dance jun drunk, chi day by day.

- Li She zhuzhi poems as basic data,

Waist drum often stick sunny ray room, dance trapped umber hang Yang liu.

- li salt use "long poetry"

Light red face small waist, fold and blowing flowers handkerchief, shook the god.

- su shi "a southern song"

Visible, slim waist, can well reflect the female's gentle, especially in the court, only amazing soft waist, can satisfy the taste of royal family. The consequent sleeve dance, sleeve technology is one of the ancient Chinese dance creation, the use of towels, sleeve, extended the human body, greatly expanded the expressive force of the body, make the emotional expression more rich and bright, towels and sleeve movement of formal beauty, can put people into a nonphysical realm. Han people $\mathrm{Fu} \mathrm{Yi} \mathrm{"dance"} \mathrm{and} \mathrm{said:} \mathrm{"the} \mathrm{clothes} \mathrm{from} \mathrm{the} \mathrm{wind,} \mathrm{long} \mathrm{sleeve} \mathrm{in} \mathrm{horizontal",} \mathrm{zhang} \mathrm{heng}$ "GuanWu fu" : "skirt fly like a phoenix, sleeve, such as back to snow" and "incense spread towels, smooth flow of jade". The use of towels, sleeve, enhances the dance skills, built the artistic conception of dance, objectively promoted the development of the dance, and dance with the intent of "good". "Better" is a kind of beyond the object, cannot be used to summarizing the concept of a "quality" and "sex", also is very difficult language to express the accurate cutting, but also can through the visual, auditory, feel to experience a kind of aesthetic interest and the intense aesthetic pleasure, "the white Zhu dance" "fairy fairy ho ying ying, xu jade bowl all coagulate if cloud line" floating zhuo about dancing; "Big he lowered his", "our clothes taste, popular with harmonized 
appearances shake" romantic and elegant, all contain "wonderful" embodies the unique size, formation of the unique artistic conception.

"And" is the ancient Chinese political ideal, is also a dance aesthetic ideal. Li zehou, liu's thought; "' and 'connotation is the unity of the perceptual desire and social ethics, is the person's psychology and spirit of unity, the unity of man and nature, individual and society, is the unity of opposite sides is the unity of the true. Nature and human society in the sense of beauty is the highest in this kind of harmonious" [3] as a result, "and" is the highest state of dance. And curled up long sleeve, slim waist, dance, 2) has been unpredictable, like clouds, like flow wave, give a person with fantasy and elegant beauty of Chinese court dance, with the so-called "long sleeve if pian-pian hong jing, fine waist curled up human" [4]. "Spring breeze fine waist get the bright moon, long sleeve dance"; [5] "curled up the waist suspected fold, Qian Qian sleeve to fly" [6] of the waist and sleeve of close cooperation, in the movement of time and space form, to renew "gets LianPian, first at first sight" [7]

" line "shape characteristic, the pursuit of a kind of" flowing "sort of" the beauty of lines ", which can create a very elegant, feminine "and" imagery, artistic conception, down to achieve dance "and" even the highest pursuit of the country's political ideal and aesthetic aspects

\section{References}

[1] B.H. Zong. The Birth of Chinese Artistic Conception. Shanghai People's Publishing House, 1981,66 .

[2] Tan-Shu T. Flying Dunhuang Art Agent . Gansu People's Publishing House, 1987, 56.

[3] Ze-Hou L, Liu's. Chinese Aesthetics (Volume 1) .China Social Sciences Press, 1984, 24.

[4] Classic Popular Library. (Yue Fu Poetry. Qing Poems. A Total of Fine Music). Yuelu Press Press, 1990, 6

[5] Xi-Yi L. Spring Female Line . Shanghai Bureau with Document Publishing House, 2002, 9.

[6] Zhang Hu. Dance Zhongzhou Ancient Books Publishing House, 2001.

[7] Zhang Heng. Guanwu Fu . Shanghai Bureau with Document Publishing House, 2002,47. 